\title{
Joint Manipulation Instruction in the Physical Therapy Curriculum of Select Philippine Higher Education Institutions
}

\author{
Lily Ann D. Bautista ${ }^{1 *}$, Andrei J. Altavas ${ }^{2}$, Cyflor E. Putong $^{1}$ and \\ Romel V. Cabazor ${ }^{3}$ \\ ${ }^{1}$ Silliman University \\ ${ }^{2}$ Physical Therapy Advancement Seminars \\ ${ }^{3}$ Velez College \\ *Iilydbautista@su.edu.ph
}

\begin{abstract}
The physical therapy graduate must possess the necessary competencies in manipulation as recommended by the World Confederation for Physical Therapy Guidelines for Entry-Level Education. This study aimed to investigate the inclusion of joint manipulation instruction in Philippine Physical Therapy Higher Education Institutions (HEIs) and determine the factors affecting its implementation. A cross-sectional descriptive survey was used to collect data and analyze findings. The Department Heads of government recognized physical therapy schools were invited to participate in the study and thirty-five schools responded to the survey. Results showed that there was limited inclusion of manipulation in the physical therapy curriculum of select Philippine HEls, with lesser application of the techniques in the spine as compared to the extremities. The respondents reported that the lack of qualified faculty in their respective institutions was the primary reason for the limited inclusion of thrust manipulation in the curriculum.
\end{abstract}

Keywords: Philippine physical therapy program, Philippine physical therapy HEl, manual therapy curriculum, manipulation, mobilization

\subsection{Introduction}

Evidence of joint manipulation techniques dates back to 460-385 BCE, when Hippocrates, the Father of Medicine, used gravity to treat scoliosis (Withington, 1928). Earlier accounts of manipulative treatments performed by European physical therapists were documented in the 1800s. Many renowned physical therapists from America, Norway, the United Kingdom, and Australia have used manipulative techniques to treat musculoskeletal patients since the 1950s. While thrust and non-thrust joint manipulation are traditionally included in the broad category of manual therapy, these techniques are not exclusive to a single profession. Manual medicine, osteopathy, and chiropractic have influenced and contributed to the diversity of the approach (Pettman, 2007). Joint manipulation provided by physical therapists has been documented in peer-reviewed journals demonstrating its efficacy and effectiveness in 
extremity musculoskeletal conditions (Bang \& Deyle, 2000; Bergman, Winters, Croesier, Pool, \& Jong, 2004; Deyle, Alison, \& Matekel, 2005; Hoeksma, Dekkar, \& Ronday, 2004; Vermeulen, Rozing, Obermann, Cessie, \& Vlieland, 2006). Clinical Practice Guidelines recommend manipulation of the spine for patients with complaints of low back pain with no neurological symptoms (Waddell, et al., 1999; Philippine Academy of Rehabilitation Medicine, 2017). Evidence shows that spinal manipulations performed by physical therapists have a low probability of causing adverse occurrences (Assendelft, Bouter, \& Knipchild, 1996; DiFabio, 1999; Rivet \& Milburn, 1997).

Joint manipulations are manual therapy techniques composed of an array of passive movements performed by skilled physical therapists to joints and soft tissues of different ranges, magnitudes, and velocities (American Physical Therapy Association, 2013). These interventions require routine and regular evaluation throughout the treatment performed by the physical therapist. Manipulation is applied to the joint within its anatomical limit to improve motion, function, and pain (International Federation of Orthopaedic Manipulative Physical Therapists, 2011). The Philippine Physical Therapy and Occupational Therapy Law supports the application of manipulation as within the scope of physical therapy practice, defining physical therapy as an "art and science of treatment utilizing therapeutic exercises, heat, cold, light, water, manual manipulation, electricity, and other physical agents" (Philippine Physical and Occupational Therapy Act of 1969). Competencies in the performance of joint manipulations are minimum required skills of physical therapy (PT) graduates in the United States (Commission on Accreditation in Physical Therapy
Education, 2017). They are considered essential in performing physical therapy treatment. This type of manual therapy is commonly subdivided into thrust and non-thrust manipulation. Thrust Manipulation (TM) is defined as a "high-velocity-low-amplitude therapeutic movement at the end range of motion" (APTA, 2013, p.2) and is typically known as joint manipulation. In contrast, Non-Thrust Manipulation (NTM) is described as a low-velocity-high-amplitude therapeutic movement, more commonly known as a joint mobilization technique. The evidence supporting joint manipulation continues to grow. The measurable effects of manipulation on a variety of musculoskeletal conditions are well documented (Masaracchio, et al., 2019; Coulter et al., 2018) and are utilized by physical therapists around the world. Interventions, including manual therapy techniques, continue to show positive results towards patient goals and outcomes (World Confederation for Physical Therapy, 2011).

The Philippine Commission on Higher Education (CHED) Memorandum Order No. 55, series of 2017, Policies, Standards and Guidelines for the Bachelor of Science in Physical Therapy (BSPT) Education, requires Higher Education Institutions (HEls) to shift educational programs towards Outcomes-Based Education. Outcomesbased model of education focuses on achieving competencies related to their field with the goal of "achieving rapid, inclusive and sustained economic growth: one that generates jobs and livelihood and increases the income of the poor" (Enhanced Basic Education Act of 2013, as cited in Sana, Roxas \& Reyes, 2015, p. 61). CMO 55, s. 2017 stipulates that HEls must adhere to its policies and procedures in the attainment of minimum requirements; however, it also encourages the HEls to establish their programs to achieve learning outcomes specific 
to its typology. There are 12 outcomes stated that are specific to the BSPT degree. The performance indicators for these outcomes cover knowledge in basic sciences; competence in evaluation, planning and implementation of physical therapy intervention; achievement of teaching and learning, communication, management, and research-related skills; promotion of health and wellness and lifelong learning; development of effective collaboration, professional and ethical responsibilities in multi-cultural settings; and utilization of current and innovative technology. One of the critical skills needed to be achieved by a PT graduate is to demonstrate competency in the application of appropriate PT intervention following a complete and sound evaluation. CMO 55 endorses the use of the World Confederation for Physical Therapy (WCPT, 2011) Guidelines for Physical Therapy Professional, Entry-Level Education. The guideline places focus on evidencebased intervention applicable across the life span. The WCPT recommends that the Bachelors' degree be at least four years in length with curriculum standards focusing on achieving knowledge, skills and attributes expected of a PT professional. Physical therapy graduates are expected to know biological and physical sciences; to have skills required to evaluate, diagnose, prognosticate and provide intervention to patients; and to possess affective behaviors and experiences in the social, behavioral, psychological and technological components. The students must possess the skills necessary to apply and use orthotic devices, physical, mechanical and electrotherapeutic modalities; and to use airway clearance, integumentary repair and protection techniques (WCPT, 2011, p. 11). They must have the competencies to perform patient instructions, therapeutic exercises, functional training, and manual therapy techniques. Manual therapy techniques may include acupressure, lymphatic drainage, massage, manual traction, passive range of motion, mobilization and manipulation (WCPT, 2011, p.25).

The instruction of joint manipulation starts in physical therapy degree programs. So far, there are no published data or information as to what extent joint manipulation is taught in Philippine physical therapy schools and how it is integrated into the curriculum. The BSPT degree is the current entrylevel point to be licensed as a Physical Therapist by the Professional Regulation Commission. The BSPT program has the required clinical sciences for teaching joint manipulation in the curriculum. Schools currently use a variety of textbooks describing the management of musculoskeletal conditions using joint manipulation in areas of the spine and peripheral joints. As joint manipulation is well documented within the scope of physical therapy practice (Philippine Physical and Occupational Therapy Act of 1969; King, 2005; CAPTE, 2017), the application of such skills should be approved by a professional body that can provide documented evidence of competence and safety.

Physical Therapists in the Philippines do not practice in a direct access environment and are dependent on referrals from physicians, primarily from the physiatrists (Rotor \& Capio, 2018). Among the recommended treatments for acute and chronic low back pain is manipulation, as outlined in the Clinical Practice Guidelines developed by PARM (2017). This shows a great need for knowledge and competency in the application of thrust manipulation in Philippine PT practice.

This research intended to survey physical therapy schools in the Philippines regarding the integration of thrust and non-thrust manipulation 
instruction in their curriculum. It is stipulated that the performance of manipulation is a required competency from a physical therapy graduate and yet there is no known research investigating its inclusion in the curriculum. The results will also determine current impediments to integration of content in the curriculum and hope to establish practice standards for joint manipulation instruction as a therapeutic intervention in the Philippine physical therapist professional degree program. Moreover, the findings will facilitate the development of a teaching model in the integration of joint manipulation in the curriculum.

\subsection{Methods}

This research is a cross-sectional descriptive survey with a quantitative approach that primarily focused on the collection of data and analysis of findings. Results were summarized to determine the status of joint manipulation instruction in physical therapy schools in the Philippines.

A convenience sample composed of Deans, Program Directors, Program Coordinators, or Department Chairpersons of CHED recognized physical therapy schools in the Philippines was utilized. The Commission on Higher Education, which is the government's agency in charge of the administration of both public and private higher education schools, listed 85 schools that offer physical therapy courses as of October 2019 on their official website. However, only 56 schools were considered due to unsuccessful attempts to gather valid contact information from the other 29 programs.

The survey tool was developed by the primary researchers with the guidance of academic professionals who supported this study. The survey questions included items addressing physical therapy program demographics, faculty member qualifications, information on joint manipulation instruction, other forms of manual therapy instruction, reasons for not teaching joint manipulation, and plans for future implementation. Five faculty members with teaching responsibilities in the BSPT program were asked to assess the survey instrument for validity, applicability, and unbiased inquiry. Face validity was assessed using a Likert Scale ranging from one (1) as strongly disagree to five (5) as strongly agree, with the results showing the five faculty members awarding a response of 4 to 5 on each of the questions. Cronbach Alpha was at 0.941 , suggesting excellent reliability. Afterward, the survey questionnaire was sent to three additional faculty members for pilot testing to ascertain that questions were clearly defined and written. The final survey instrument underwent minor revisions based on the feedback received.

The definitions of both thrust and non-thrust manipulation were included in the survey tool to ensure a clear understanding of the terminologies.

\section{Survey Administration}

The Silliman University Ethics Board approved the study protocol. Four weeks before sending the survey, contact information including email and phone number of each school representative were gathered. The survey packet, which included the questionnaire and a cover letter describing the study's purpose and objectives, was sent to 56 select HEls. The cover letter emphasized that results would be reported with the anonymity of individual responses and that participation was voluntary. Follow-up attempts were made to contact the representatives through phone calls or text messages when the survey was unreturned. 
All survey responses were tracked and coded from October 2019 through February 2020.

\section{Data Analysis}

The survey responses were collected and entered into an Excel spreadsheet (Microsoft Corp., Redmond WA) by one of the researchers. The data were then independently reviewed by another investigator of the study. Descriptive statistics were calculated to determine the demographics of physical therapist schools, faculty qualifications, and the status of joint manipulation instruction in Philippine PT schools.

\subsection{Results \& Discussion}

\section{School and Faculty Demographics}

A total of 35 schools (62.5\%) from the following regions: 22 Luzon, 9 Visayas, 4 Mindanao responded to our survey (Figure 1). Thirty-five of the surveyed schools offered a Bachelor of Science in Physical Therapy program. Master of Rehab Science (MRS) and Master of Science in Physical Therapy (MSPT) are also offered in two and five of the thirtyfive schools, respectively (Figure 2). Twenty-six schools (74.2\%) indicated that a Master's degree was required to teach in their physical therapy program, while nine schools (25.7\%) required a Bachelor's degree to be a faculty member (Figure 3 ).

Majority of the schools (77.1\%) engaged in faculty team teaching, while the minority $(22.8 \%)$ had a dedicated faculty for each course (Figure 4). As for the professional preparation, 15 schools $(42.8 \%)$ indicated that their faculty received a postprofessional manual therapy training from various philosophies (Somatic, Osteopathic, mixed), and did not require teaching or previous clinical experience to teach manual therapy.

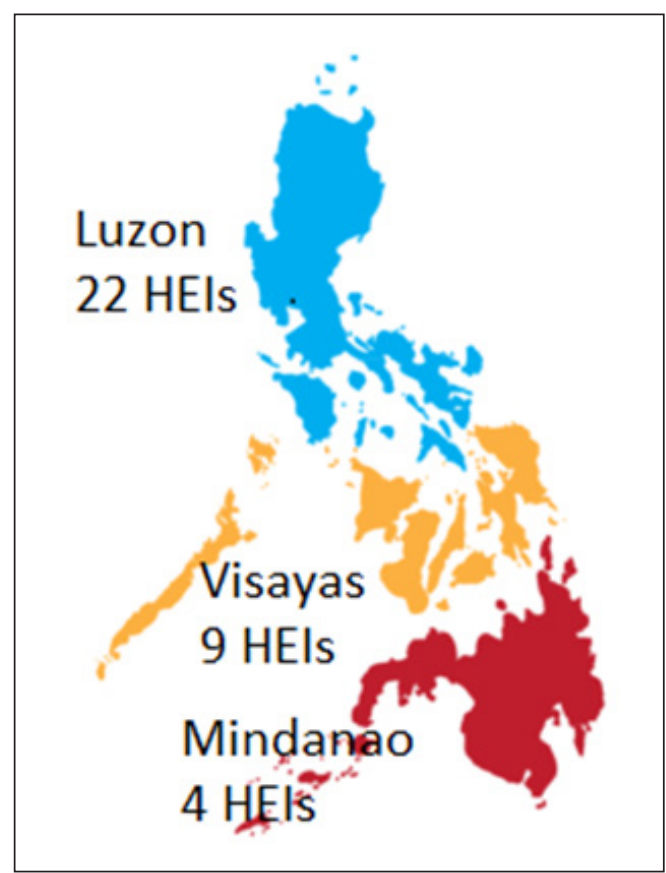

Figure 1. Regional Distribution of HEl Respondents 


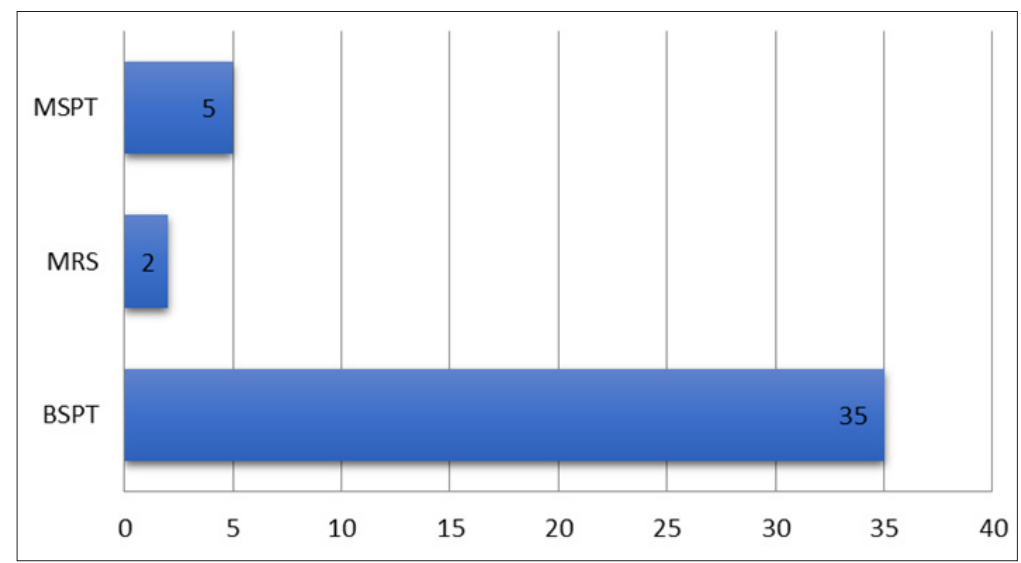

Figure 2. Program Offering of Surveyed Schools

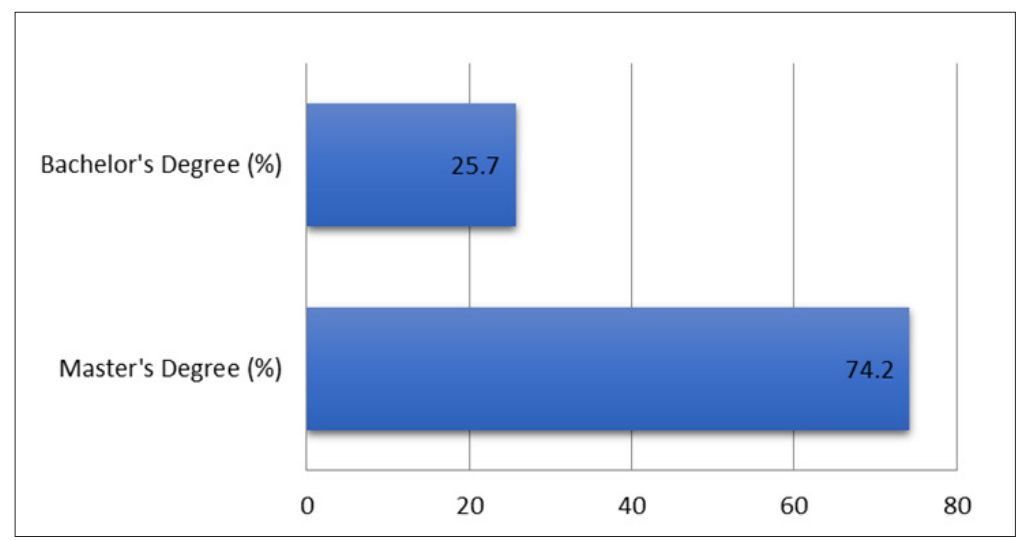

Figure 3. Faculty Credential Requirement of Surveyed Schools

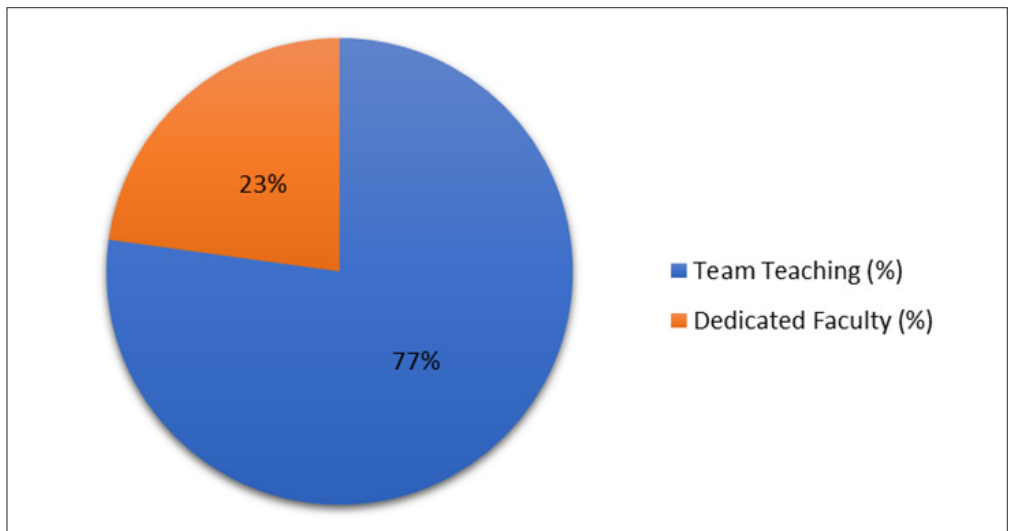

Figure 4. Teaching Method of Surveyed Schools 


\section{Joint Manipulation Instruction}

Table 1. Summary of PT Schools Teaching Joint Manipulation

\begin{tabular}{lcc}
\hline Techniques & $\mathbf{n}$ & \% \\
\hline Non-Thrust Manipulation & 31 & 88.5 \\
Thrust Manipulation & 13 & 37.1 \\
"Other" Manual Therapy & 35 & 100 \\
\hline
\end{tabular}

Table 2. Joint Manipulation Instruction by Body Region

\begin{tabular}{lcc}
\hline Body Region & Non-Thrust (\%) & Thrust (\%) \\
\hline Extremities & 94.2 & 31.4 \\
Spine & 60 & 11.4 \\
\hline
\end{tabular}

Non-thrust manipulation and TM were part of the curricular content of the 35 schools, $88.5 \%$ and $37.1 \%$, respectively (Table 1 ). Relating to the body region where joint manipulation is taught,
$94.2 \%$ of the schools reported instructions on NTM techniques of the extremities, whereas only $60 \%$ reported instructions on spine techniques (Table 2). Conversely, TM techniques were taught on the extremities and spine, amongst $31.4 \%$ and $11.4 \%$ of the respondents, respectively (Table 2). All respondents also included manual therapy instruction in the form of soft tissue mobilization and/or muscle energy technique.

The schools currently not teaching joint manipulation reported reasons due to lack of qualified faculty $(71.4 \%)$ and belief $(17.1 \%)$ that it was not appropriate for BSPT students (Figure 5). Respondents described plans to include TM in their curriculum by initiating a new course in TM (22.8\%), modify existing courses to include TM (28.5\%), send faculty for training (91.4\%), and the remaining schools (8.5\%) were undecided (Figure 6). Finally, eighty percent $(80 \%)$ of respondents stated they were aware that joint manipulation was included as an intervention under the Philippine Physical Therapy and Occupational Therapy Law (RA 5680).

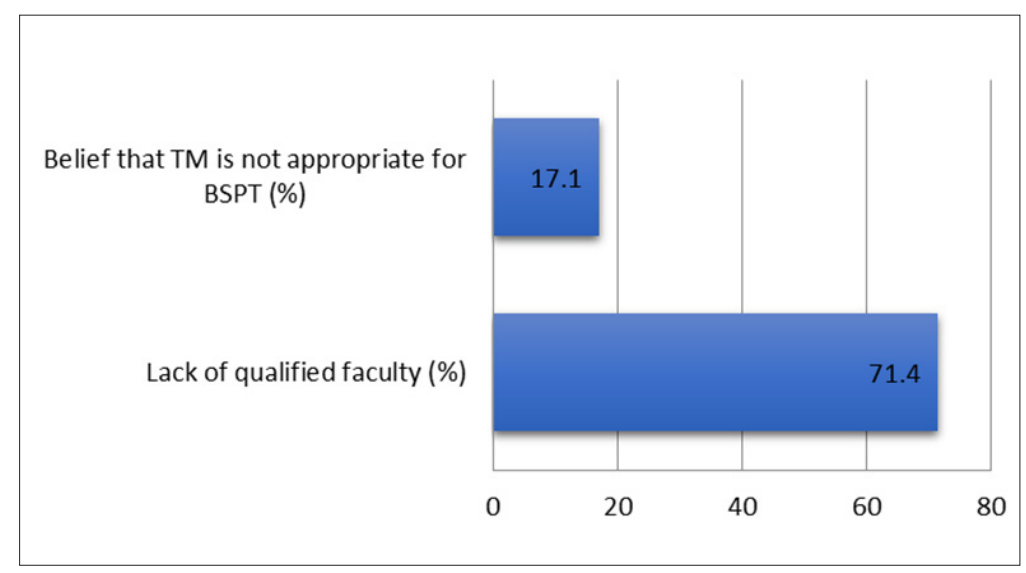

Figure 5. Reasons for Not Teaching Thrust Manipulation (TM) 


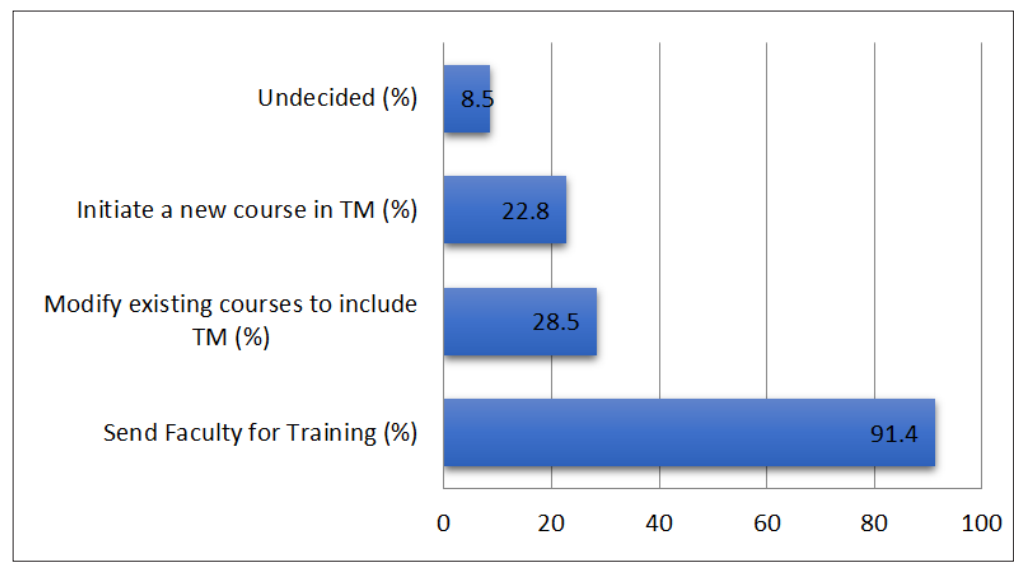

Figure 6. Programs' Plans for TM Instructions

This survey is the first to investigate the inclusion of joint manipulation in the physical therapy curriculum of select Philippine HEls. The results showed evidence of NTM instruction primarily in the peripheral joints. The study revealed the lack of TM instructions, particularly in the spine region. We anticipated the number of schools teaching NTM to be in this range due to existing textbooks used in entry-level PT programs covering such techniques. In this regard, it was sensible to focus our discussion on the survey results concerning TM instruction.

Earlier investigation on teaching trends showed that TM is learned through post-professional educational activities, including continuing education seminars and certification programs (Boissonnault, Bryan, \& Fox, 2004; Boissonault, Noteboom, \& Little, 2012). In other countries like the United States, manipulation instruction can be found in nearly all entry-level Doctor of Physical Therapy (DPT) programs (Boissonnault et al., 2012). Additionally, the APTA (2009) stated in their position statement on thrust manipulation that training should start in the entry-level PT program. Although the entry-level degree in the United States is a clinical doctorate level compared to the BSPT program in the Philippines, it is worthwhile noting that the Philippine PT curriculum is comparable to that of the DPT curriculum (Texas Tech University Health Sciences Center, 2020; A.T. Still University, n.d.; CMO 55, s. 2017). Therefore, this should not preclude undergraduate programs (e.g., BSPT) in adopting a similar integration of thrust manipulation instruction in the curriculum. Whether this model is adopted by Philippine standards remains a subject for future policy.

Furthermore, the results could identify existing barriers to effectively integrate thrust and non-thrust manipulation into the PT curriculum and provide direction for the academic community for faculty resources and opportunities. Based on our data, the primary reason for schools not teaching TM was due to the lack of faculty training and exposure to the technique. The researchers also believe that postgraduate education is vital. CHED (CMO 26, s. 2009) sought to upgrade the knowledge of all faculty members to masters or doctoral level, to ensure delivery of quality education. It is stipulated in CMO 55, s. 2017, that faculty members teaching PT professional subjects must be at least enrolled in 
a master's program, and yet our research found that $25.7 \%$ of the HEI respondents only require a BSPT degree.

Safe and effective application of TM begins in the school, where clinical reasoning skills are developed (Corkery, Hensley, Cesario, Yen, \& Courtney, 2020). Many of the NTM (or mobilization) techniques can easily be converted into thrust techniques by adding speed. This skill can be taught at a basic level of instruction. For instance, when students perform passive range of motion, adding a joint glide or arthrokinematics motion becomes a non-thrust technique and progression to a high-velocity-low-amplitude movement turns the procedure into a thrust maneuver. An understanding of anatomy, kinesiology and biomechanics with clinical rationale is required to perform manipulation with a subsequent examination to modify methods in the treatment session (Olson, 2016). While we cannot disagree with the fact that faculty training is mandatory, the application of techniques is rooted in the clinical decision-making guidance provided to BSPT students. This, along with supervised clinical education during internship, prepares the student to safely utilize manipulative procedures. We agree that the detailed neuromusculoskeletal examination process physical therapists learn is more suitable for post-professional residency or postgraduation clinical education. Only 15 schools (42.8\%) have teaching staff with post-professional training in manual therapy, despite the results showing that $88.5 \%$ and $37.1 \%$ of the schools teach, NTM and $\mathrm{TM}$, respectively. Due to the lack of previous studies investigating the trends in post-professional manual therapy training in the Philippines, we are unable to give a plausible explanation behind this data.

The lack of formal post-professional training in TM may result in a school not having faculty with the appropriate skills to teach thrust manipulation.
Our study did not investigate the extent of courses in the PT curriculum, where joint manipulation is taught. Previous studies have reported a variation on how joint manipulation was integrated into the physical therapist curriculum and Boissonnault et al. $(2004,2012)$ found that manipulation instruction was generally part of the clinical science courses and a component of multiple classes in the United States Physical Therapist programs. Knowledge in general sciences (anatomy, kinesiology, physiology) and clinical sciences (medical/surgical, pathology, therapeutic exercise), necessary to develop competencies and skills in joint manipulation, are included in the entry-level Philippine BSPT curriculum (CMO 55, s. 2017). And yet, manipulation instructions, particularly TM are not fully integrated into the curriculum

The results of our survey indicate a strong need for post-professional training in joint manipulation for physical therapy faculty. The study could serve as a criterion for a review of current physical therapy courses and determine how joint manipulation can be integrated into the curriculum. Ninety-one percent of physical therapist programs indicated plans to send faculty for training if supported by a professional body or if school administration supports the cost. If the goal is to prepare students to be efficient and effective clinicians upon graduation, schools need to teach techniques that are essential in musculoskeletal physical therapy care.

\section{Limitations}

Our survey generated a response rate of $62.5 \%$, which is within the range of acceptability based on the number of survey packets sent out $(n=56)$. Eighty-five schools were listed as physical therapist programs under the Commission on 
Higher Education (CHED). We made every effort to contact schools listed on the CHED website, but it was likely that the contact information of some schools was outdated. The response rate would have been significantly below the range of generalizability (41.1\%) if the total number of CHED schools were surveyed. Luzon region includes the national capital $(n=60)$ and has a higher number of long-established programs than schools in the Visayas $(n=12)$ and Mindanao $(n=13)$ regions of the Philippines.

It should be noted that Silliman University and Velez College, both from the Visayas region, are proponents of this study, therefore, excluded from the research. Many physical therapist degree programs are offered through Colleges and Universities, each having different academic emphasis (research vs. teaching; public vs. private; stand-alone vs. medical center-based; small vs. large). It is also possible that some schools have not yet produced physical therapy graduates or are in the process of undergoing accreditation. This information was not available during our initial sampling. Lastly, our survey did not investigate the educational program's focus that could explain the variations in manual therapy instruction.

\subsection{Conclusion}

Thirty-five select Physical Therapy HEls in the Philippines provided us preliminary data on the inclusion of joint manipulation instruction in their curriculum. Most schools surveyed did not teach joint manipulation in their program due to a lack of faculty training or exposure. There is a lack of compliance with regards to faculty qualification, as stipulated in CMO 55, s. 2017, with some of the school respondents. The results also demonstrated a predominance of other types of manual therapy interventions. Joint manipulation was included in the physical therapy curriculum of $37.1 \%$ select Philippine HEls.

The need for opportunities for postprofessional faculty training in joint manipulation or a clinical postgraduate degree is proposed to ensure the delivery of quality physical therapy education. Further studies are warranted to determine the extent of administrative support for postgraduate faculty training and to study the possible consequences of non-compliance to regulatory standards regarding the inclusion of joint manipulation instruction and faculty training and qualifications.

\section{Acknowledgments}

The authors are thankful to Mr. Bernd Haft, Silliman University PT Intern, and Mr. King Ricafort, PTRP, Silliman University alumnus, for their valuable assistance in data gathering.

\section{References}

American Physical Therapy Association, (2009). Position on thrust manipulation provided by Physical Therapists. https://www.apta.org/ uploadedFiles/APTAorg/Advocacy/State/Issues/ Manipulation/WhitePaperSummaryManipulation. pdf

American Physical Therapy Association (2013). Physical therapists and direction of mobilization/ manipulation: An educational resource paper. http://www.apta.org/Statelssues/Manipulation/ PTsDirectionofMobilizationManipulation

Assendelft, W.J.J., Bouter, L.M., \& Knipschild, P.G. (1996). Complications of spinal manipulation. 
A comprehensive review of the literature. The Journal of Family Practice, 42(5), 475-480.

A.T. Still University (n.d.). Doctor of physical therapy degree. https://www.atsu.edu/doctor-of-physicaltherapy-degree\#curriculum

Bang, M.D. \& Deyle, G.D. (2000). Comparison of supervised exercise with and without manual physical therapy for patients with shoulder impingement syndrome. JOSPT, 30(3), 126137.

Bergman, G.J., Winters, J., Croesier, K.H., Pool, JM, \& Jong, B (2004). Manipulative therapy, in addition to usual medical care for patients with shoulder dysfunction and pain: a randomized controlled trial. Ann Intern Med, 141(6), 432-9.

Boissonnault, W., Bryan, J.M., \& Fox, K.J (2004). Joint manipulation curricula in physical therapist professional degree programs. J Orthop Sports Phys Ther, 34, 171-178.

Boissonnault, W., Noteboom, J.T., \& Little, C. (2012) Thrust Joint Manipulation Curricula in FirstProfessional Physical Therapy Education: 2012 Update. J of Ortho Sports Phys Ther, 45(6), 471-476.

Commission on Accreditation in Physical Therapy Education, (2017). Standards and required elements for accreditation of physical therapist education programs. http://www.capteonline. org/uploadedFiles/CAPTEorg/About_CAPTE/ Resources/Accreditation_Handbook/CAPTE_ PTStandardsEvidence.pdf
Commission on Higher Education (2009). Revised implementing guidelines of the commission on higher education-faculty development programs (CHED-FDPs) Phase 2 (CMO 26, s. 2009). https://ched.gov.ph/wp-content/ uploads/2017/10/CMO-No.26-s2009-1.pdf

Commission on Higher Education (2017). Policies, standards and guidelines for the bachelor of science in physical therapy (BSPT) education (CMO 55, s. 2017). https://ched.gov.ph/cmono-55-series-of-2017-policies-standards-andguidelines-for-the-bachelor-of-science-inphysical-therapy-bspt-education/

Corkery, M.B., Hensley, C.P., Cesario, C., Yen, S., \& Courtney, C. (2020). Use of thrust joint manipulation by student physical therapists in the United States during clinical education experiences. J Man ManipTher.

Coulter, I.D., Crawford, C., Hurwitz, E.L., Vernon, H., Khorsan, R., Booth, M.S., \& Herman, P.M. (2018). Manipulation and mobilization for treating chronic low back pain: a systematic review and meta-analysis. Spine, 18(5), 866879.

Deyle, G.D., Allison, S.C., \&Matekel, R.L. (2005). Physical therapy treatment effectiveness for osteoarthritis of the knee: a randomized comparison of supervised clinical exercise and manual therapy procedures versus a home exercise program. Phys Ther, 85(12), 1310-1317.

DiFabio, R.P. (1999). Manipulation of the cervical spine: risks and benefits. Phys Ther, 79(1), 50-65. 
Hoeksma, H.L., Dekkar, J., \&Ronday, H.K. (2004). Comparison of manual therapy and exercise in osteoarthritis of the hip: a randomized clinical trial. Arthritis and Rheumatism, 51(5), 722-729.

King, P.M. (2005). The Manipulation Education Manual: Support for Evidence-Based Education [Editorial]. Journal of Orthopaedic \& Sports Physical Therapy, 35(7).

International Federation of Orthopedic Manipulative Therapists, (2011). Educational Standards: A Historical Perspective. https://www.ifompt. org/site/ifompt/files/pdf/IFOMPT\%20 History\%20definitive\%202011\%20_4_.pdf

Masaracchio, M., Kirker, K., States, R., Hanney, W., Liu, X., \&Kolber, M. (2019). Thoracic manipulation for the management of mechanical neck pain: A systematic review and meta-analysis. PLOS ONE, 14(2), e0211877.

Olson, K. (2016). Manual physical therapy of the spine, 2nd Ed. Elsevier. St. Louis, MO.

Pettman, E. (2007). A history of manipulative therapy. The Journal of Manual and Manipulative Therapy, 16(3), 165-174.

Philippines.1969. Republic Act no. 5680: An Act Creating the Board of Examiners for Physical Therapists and Occupational Therapists. https://www.thecorpusjuris.com/legislative/ republic-acts/ra-no-5680.php

Philippine Academy of Rehabilitation Medicine, (2017). Clinical Practice Guidelines on Diagnosis and Management of Low Back Pain
(Updated:2017). http://parm.com.ph/clinicalpractice-guidelines/

Rivett, D.A., \& Milburn, P. (1997). Complications arising from spinal manipulative therapy in New Zealand. Physiotherapy, 83(12), 626-632.

Rotor, ER, \&Capio, C.M. (2018). Clinical reasoning of Filipino physical therapists: Experiences in a developing nation. Physiotherapy Theory and Practice, 34(3), 181-193.

Sana, E. A., Roxas, A.B., \& Reyes, A.L.T. (2015). Introduction of outcome-based education in Philippine health professions education setting. Philippine Journal of Health Research and Development, 19(1), 60-74.

Texas Tech University Health Sciences Center (2020). Doctor of physical therapy curriculum. https:// www.ttuhsc.edu/health-professions/doctorof-physical-therapy/curriculum.aspx

Vermeulen, H.M., Rozing, P.M., Obermann, W.R., Cessie, S.L., \&Vlieland, TPMV (2006). Comparison of high-grade and low-grade mobilization techniques in the management of adhesive capsulitis of the shoulder: randomized controlled trial. Phys Ther, 86(3), 355-368.

Waddell, G., Mclntosh, A., Hutchinson, A., Feder, M.,\& Lewis, M. (1999). Low back pain evidence reviews. London, UK: Royal College of General Practitioners.

Withington, E.T (1928). Hippocrates. With an English Translation. Cambridge, MA: Harvard University Press. 
World Confederation for Physical Therapy (2011). WCPT guideline for physical therapist professional entry-level education. https:// www.wcpt.org/sites/wcpt.org/files/files/ Guideline_PTEducation_complete.pdf 